\title{
The Study on the Change Rules of Drive Motor Load Based on the Vehicle Vibration Model
}

\author{
Yuqing Cui*, Xiaobo Qiu and Chen Zhang \\ Academy of Armored Force Engineering, Beijing, 100072, P. R. China \\ ${ }^{*}$ Corresponding author
}

\begin{abstract}
This article give the rules of changing torque which produced in drive system during the movement by modeling and simulating the vibration of vehicle, and try to have a meaningful guiding significance for the motor selection and control. First, the article analyzes the reason that vibration affect motor load, then models and simulates the road roughness and vehicle vibration, finally gets the changing rules of the drive motor load. The result turns out: the vibration can make the motor load for an additional $24 \%$ on average, at some point even reach $90 \%$.
\end{abstract}

Keywords- road roughness; vehicle vibration; motor load

\section{INTRODUCTION}

During the vehicle movement, because of the existence of the dive system backlash, the transmission accuracy of drive system is low and can't satisfy the need of the actual situation. It is becoming more and more necessary to transform the present dive system to make them have the high transmission accuracy. After a careful analysis, we put forward a solution, using double-motor anti-backlash system to transform the original drive system, to make the vehicle have accurate transmission ability under the condition of high mobility. In this plan, because the movement can make the vehicle have inevitable vibration, the vibration produce a constantly changing torque which acting on drive system. Combined with the traditional worm wheel transmission mechanism can't fit with double-motor system ${ }^{[1]}$, all this changing torque only offset by the torque which provided from the driving motor. So this transform plan comes up with a more complex test on the selection and control of motors. This article is trying to model and simulate the changing torque which produced in drive system during the movement so that can have a useful meaning for motor selection and control.

\section{SimPlified MechaniCAL MOdEL FOR DRIVE SYSTEM}

The embodiment of the drive system transmission accuracy is through the turning of elevating arc to embody. The elevating arc which is rigid connect with the trunnion is driven by drive gears which is driven by motors through the reducers. When the vehicle is on the move, we approximately regard drive gears and trunnion as rigid connect with the vehicle body, they will have the same vibration compared with the body. In double-motor system, to control elevating arc at any time, the drive gears must have an extra torque acting on the elevating arc to keep it having the same movement with the vehicle body. Only in this way, can we make sure that the drive system is in a high transmission accuracy all the time. Through the stress analysis of the drive gears, we study the magnitude of the torque that motors should provide.

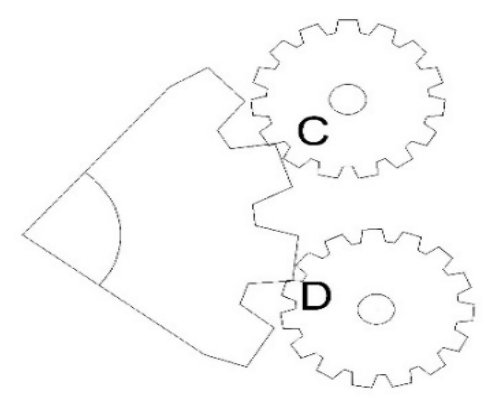

FIGURE I. DIVE GEARS AND ELEVATING ARC MESHED

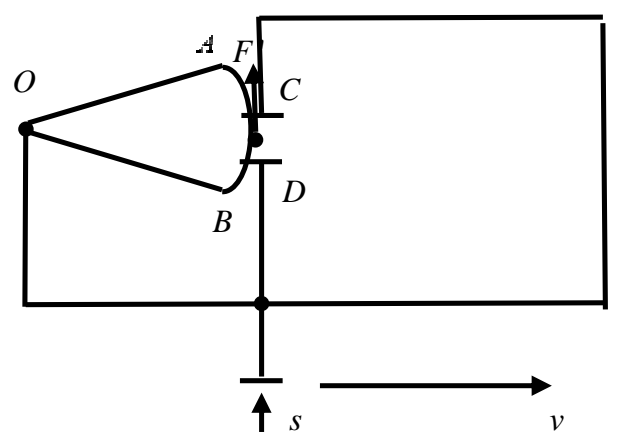

FIGURE II. SIMPLIFIED MECHANICAL MODEL FOR DRIVE SYSTEM

From figure II, $O$ is the position of trunnion, $C 、 D$ are the mesh points of double drive gears and elevating arc, $O A B$ is the elevating arc, $F$ is applied force to drive gears provide by elevating arc, $V$ is the velocity of the vehicle, $S$ is the elevation vibration displacement of the vehicle.

According to figure II, we can easily know that two drive gears should at least provide the force as the same size as the 
$F$ to maintain the arc vibrate with the vehicle synchronously. $F$ is directly associated with the condition of the vehicle vibration, especially about the vehicle body vibration acceleration, so it's necessary to figure out the laws of the vehicle vibration.

\section{Model AND Simulation OF THE Vehicle VibRation}

The reason of vehicle vibration is that the road is uneven, the uneven road will cause the tires vibrating. Although this vibration can lighten a lot through suspension system, it is still affect the vehicle body and make it vibrate. So road is the key factor which causes vehicle vibration, so simulating road surface irregularity is the most important in vehicle dynamic simulation.

\section{A. Road Roughness}

Road roughness is used to describe the level of the uneven road in the research of the road engineering. Road roughness is the road surface's degree of deviation to the ideal surface, it can affect the vehicles' power performance, roadway 's running quality and road dynamic loading[2]. According to GB/T7031-2005/SO8608: 1995'’Mechanical vibration-Road surface profiles-Reporting of measured data', road roughness' power spectral density (PSD) fitting expression is:

$$
G_{q}(n)=G_{q}\left(n_{0}\right)\left(\frac{n}{n_{0}}\right)^{-\omega}
$$

Where $n$ is spatial frequency, and it's physical meaning is the reciprocal of signal wavelength; $n_{0}$ is reference frequency, usually $n_{0}=0.1 \mathrm{~m}^{-1} ; \omega$ is frequency index, $\omega=2 ; G_{q}\left(n_{0}\right)$ is the PSD in reference frequency, it is also called road roughness index.

Because of the differences of the road roughness index $G_{q}\left(n_{0}\right)$, International Standard Association divides the road surface into 8 levels, and give the corresponding parameter values of the every road surface level, its details are showed in TABLE I, $\sigma_{q}^{2}$ is the variance of road surface.

TABLE I. THE LEVEL OF ROAD SURFACE

\begin{tabular}{|c|c|c|c|c|c|c|}
\hline \multirow{2}{*}{$\begin{array}{l}\text { Road } \\
\text { level }\end{array}$} & \multicolumn{3}{|c|}{$G_{q}\left(n_{0}\right)\left(\times 10^{-6} \mathrm{~m}^{2} / \mathrm{m}^{-1}\right)$} & \multicolumn{3}{c|}{$\sigma_{q}^{2}\left(\times 10^{-3} \mathrm{~m}\right)$} \\
\cline { 2 - 7 } & $\min$ & aver & $\max$ & $\min$ & aver & $\max$ \\
\hline A & 8 & 16 & 32 & 2.69 & 3.81 & 5.38 \\
\hline B & 32 & 64 & 128 & 5.38 & 7.61 & 10.77 \\
\hline C & 128 & 256 & 512 & 10.77 & 15.23 & 21.53 \\
\hline
\end{tabular}

\begin{tabular}{|c|c|c|c|c|c|c|}
\hline D & 512 & 1024 & 2048 & 21.53 & 30.45 & 43.06 \\
\hline E & 1024 & 4096 & 8192 & 43.06 & 60.90 & 86.13 \\
\hline F & 8192 & 16384 & 32768 & 86.13 & 121.80 & 172.26 \\
\hline G & 32768 & 65536 & $\begin{array}{c}13107 \\
2\end{array}$ & $\begin{array}{c}172.2 \\
6\end{array}$ & 243.61 & 344.52 \\
\hline H & $\begin{array}{c}13107 \\
2\end{array}$ & $\begin{array}{c}26214 \\
4\end{array}$ & $\begin{array}{c}52428 \\
8\end{array}$ & $\begin{array}{c}344.5 \\
2\end{array}$ & 687.22 & 689.04 \\
\hline
\end{tabular}

B. Simulation of the Road Roughness

Besides the effects of the road roughness, the vehicle vibration is also related with the vehicle velocity. When the vehicle drive on the road, which $n$ is its spatial frequency, with the velocity $V$, the relationship between the input temporal frequency and spatial frequency is:

$$
f=v n
$$

Because of that, the temporal frequency PSD within a certain velocity is:

$$
G_{q}(f)=\frac{1}{v} G_{q}(n)
$$

In this article, we generate the random road roughness by the PSD based on trigonometric series method[3]. Assuming that temporal frequency in the range of $\left(f_{1}, f_{2}\right)$, according to temporal frequency PSD, use the deployment specification of the average power spectra, the road roughness variance is:

$$
\sigma^{2}=\int_{f_{1}}^{f_{2}} G_{q}(f) d f
$$

$\left(f_{1}, f_{2}\right)$ is divided into $n$ subintervals, make the density value $G_{q}\left(f_{\text {mid-i }}\right)$, which is the density value of each subinterval's center frequency $f_{\text {mid-i }}(\mathrm{i}=1,2, \ldots, \mathrm{n})$, as the value of this subinterval:

$$
\sigma^{2} \approx \sum_{i=1}^{n} G_{q}\left(f_{\text {mid }-i}\right) \cdot \Delta f_{i}
$$

Assume that:

$$
A_{i}=\sqrt{G_{q}\left(f_{\text {mid }-i}\right) \cdot \Delta f_{i}}
$$

Because of the standard deviation of sinusoidal signal $\sqrt{2} A_{i} \sin \left(2 \pi f_{\text {mid }-i} t+\theta_{i}\right)$ equaling to $A_{i}$, we can get road 
random roughness $q(t)$ in the time domain by superposing $n$ such sinusoidal signals:

$$
q(t)=\sum_{i=1}^{n} \sqrt{2 G_{q}\left(f_{\text {mid-i }}\right) \Delta f_{i}} \cdot \sin \left(2 \pi f t-\theta_{i}\right)
$$

Combine with (2), (3), (7):

$$
q(t)=\sum_{i=1}^{n} \sqrt{2 G_{q}\left(n_{\text {mid-i }}\right) \Delta n_{i}} \cdot \sin \left(2 \pi v n t-\theta_{i}\right)
$$

Where ${ }^{\theta_{i}}$ is the random number in the range of $[0,2 \pi]$; $V$ is vehicle's velocity; $t$ is drive time; $\Delta n$ is temporal frequency increment, $\Delta n=0.005 ; G_{q}\left(n_{\text {mid-i }}\right)$ is PSD.

For example, the vehicle at the speed of $25 \mathrm{~km} / \mathrm{h}$ on the $\mathrm{D}$ level road has road roughness condition as showed in FIGURE III:

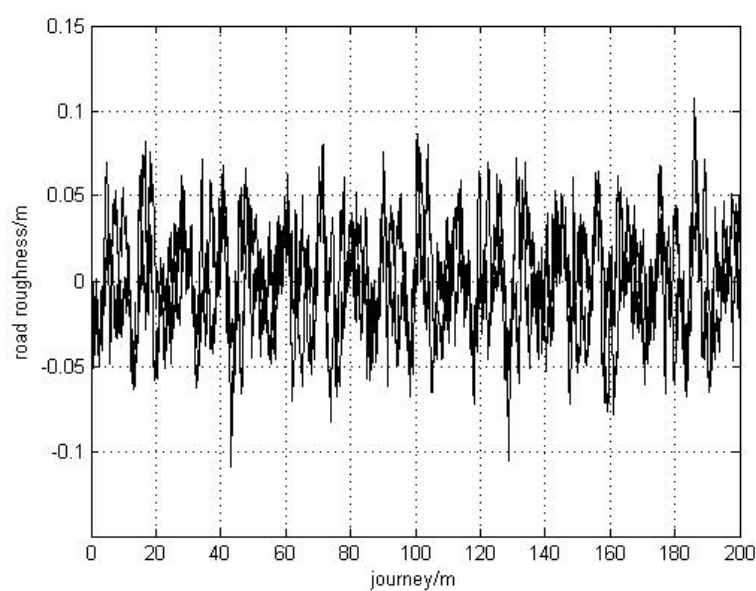

FIGURE III. ROAD ROUGHNESS ON THE D LEVEL ROAD AT THE SPEED OF $25 \mathrm{KM} / \mathrm{H}$

\section{Model of the Vehicle Vibration}

Because this article research emphasis is not on the analysis of the car body vibration rule, the simulation of vehicle vibration model does not need a detailed analysis. Now regard the vehicle as a single round double mass two degree of freedom model:

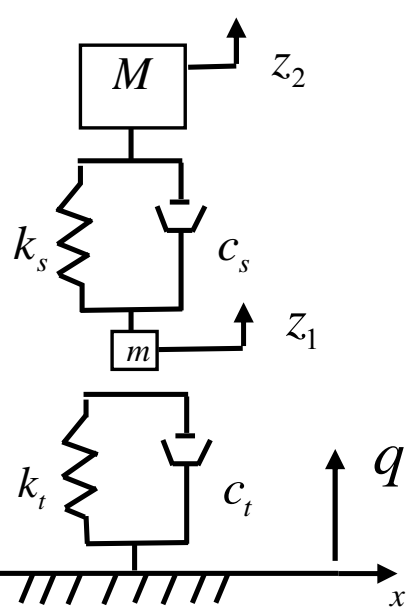

FIGURE IV. SINGLE ROUND DOUBLE MASS TWO DEGREE OF FREEDOM MODEL

where $M$ is the quality of the vehicle body; $m$ is the quality of the tires; ${ }^{s}$ is the rigidity of the suspension system; $c_{s}$ is the damping of the suspension system; ${ }^{t}$ is the rigidity of the tires; ${ }^{C_{t}}$ is the damping of the tires; ${ }^{Z_{1}}$ is the absolute displacement of the tire center; ${ }^{Z}$ is the absolute displacement of the vehicle body; $q_{\text {is the road roughness. }}$ is:

The equation of motion of damped forced vibration system

$$
m \ddot{z}(t)+c \dot{z}(t)+k z(t)=k_{t} q(t)
$$

In combination with the practical situation of the model, obtain the differential equation:

$$
\left\{\begin{array}{c}
M \ddot{z_{2}}+c_{s}\left(\dot{z_{2}}-\dot{z_{1}}\right)+k_{s}\left(z_{2}-z_{1}\right)=0 \\
\ddot{m} \ddot{z}_{1}+c_{s}\left(\dot{z_{1}}-\dot{z_{2}}\right)+c_{t} \dot{z}_{1}+k_{s}\left(z_{1}-z_{2}\right)+k_{t} z_{1}=k_{t} q
\end{array}\right.
$$

$$
\begin{aligned}
& \quad X=\left[\begin{array}{cccc}
\dot{z_{2}} & z_{2} & \dot{z_{1}} & z_{1}
\end{array}\right]^{T} \\
& Y=\left[\begin{array}{ccc}
\ddot{z_{2}} & \dot{z_{2}} & z_{2}
\end{array}\right]^{T} \text {, obtain the equation of state: }
\end{aligned}
$$

$$
\left\{\begin{array}{l}
\dot{X}=A X+B u \\
Y=C X+D u
\end{array}\right.
$$


Where

$$
\begin{aligned}
A & =\left[\begin{array}{cccc}
\frac{C_{s}}{M} & \frac{k_{s}}{M} & -\frac{C_{s}}{M} & -\frac{k_{s}}{M} \\
1 & 0 & 0 & 0 \\
-\frac{C_{s}}{m} & -\frac{k_{s}}{m} & \frac{C_{s}+C_{t}}{m} & \frac{k_{s}+k_{t}}{m} \\
0 & 0 & 1 & 0
\end{array}\right] \\
B & =\left[\begin{array}{cc}
0 & 0 \\
0 & 0 \\
\frac{k_{t}}{m} & \frac{C_{t}}{m} \\
0 & 0
\end{array}\right], \quad C=\left[\begin{array}{cccc}
\frac{C_{s}}{M} & \frac{k_{s}}{M} & -\frac{C_{s}}{M} & -\frac{k_{s}}{M} \\
1 & 0 & 0 & 0 \\
0 & 1 & 0 & 0
\end{array}\right], \\
D & =0, u=\left[\begin{array}{c}
q \\
\dot{q}
\end{array}\right] .
\end{aligned}
$$

\section{Simulation of the Vehicle Vibration}

Put the road roughness into the model of the vehicle, when the vehicle drive on the D level road at the speed of $25 \mathrm{~km} / \mathrm{h}$, the body vibration condition is showed as follow:

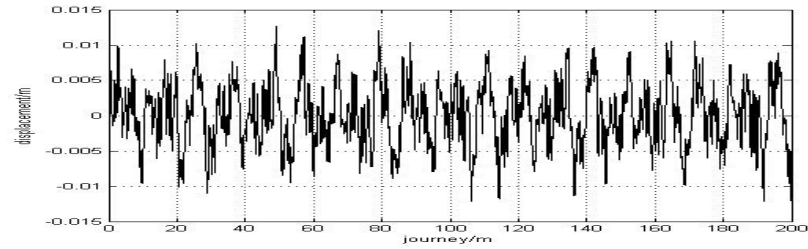

FIGURE V. VEHICLE BODY VIBRATION DISPLACEMENT VARIATION

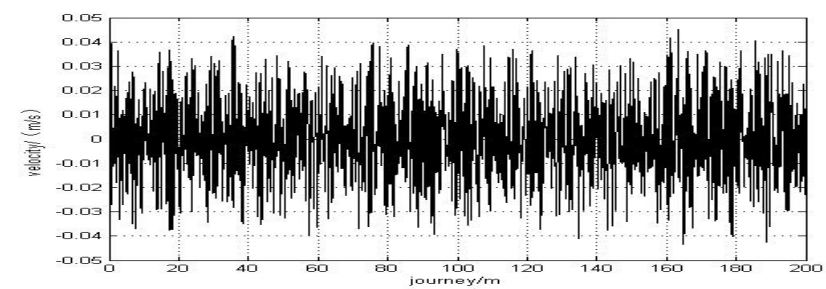

FIGURE VI. VEHICLE BODY VIBRATION VELOCITY VARIATION

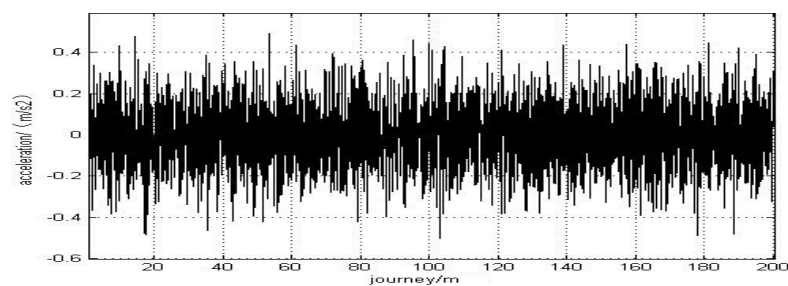

FIGURE VII. VEHICLE BODY VIBRATION ACCELERATION VARIATION
From the figures, the average of the vehicle body vibration displacement absolute value is $0.0045 \mathrm{~m}$; the average of the vehicle body vibration velocity absolute value is $0.016 \mathrm{~m} / \mathrm{s}$, the average of the vehicle body vibration acceleration absolute value is $0.144 \mathrm{~m} / \mathrm{s} 2$. In the meantime, the maximum of the vehicle body vibration displacement absolute value is $0.013 \mathrm{~m}$, the maximum of the vehicle body vibration velocity absolute value is $0.045 \mathrm{~m} / \mathrm{s}$, the maximum of the vehicle body vibration acceleration absolute value is $0.5 \mathrm{~m} / \mathrm{s} 2$.

\section{CONCLUSION}

Through the above modeling and simulation, we can find out the rule of the vehicle vibration in the process of the movement. After the calculation, compared with parameter in the quiescent state, the vibration will make the motor load for an additional $24 \%$ on average, at some point even reach $90 \%$. Through the process of modeling and simulation of the vehicle body vibration, this article gets the vehicle disturbance torque variation law in the process of movement and provides a meaningful reference significance for the motor selection and control of the double-motor drive system.

\section{REFERENCES}

[1] Tianhua Lai, "The design and practice of high precision servo drive system," Electro-Mechanical Engineering, vol. 4, pp. 46-53, 1996.

[2] Huming Duan, Feng Shi, Fei Xie, Kaibin Zhang, "The research overview of road roughness," Journal of Vibration and Shock, vol. 28, pp. 95-101, 2009.

[3] Zhaoxin Xu, "Random Vibration," Beijing: Higher Education Press, 1990.

[4] Wenbo Bao, Quan Bai, Haiyan Lu, "Vibration mechanics foundation and MATLAB Application,” Beijing: Tsinghua University Press, 2015 